\title{
La realidad como mito: el contexto de las universidades ante la complejidad posmoderna
}

\author{
Reality as a myth: the context of universities and the \\ postmodern complexity
}

DOI: https://doi.org/10.32870/dse.v0i22.910

\section{Guillermo Isaac González Rodríguez*}

\begin{abstract}
Resumen
Ante las diversas problemáticas derivadas de la pandemia de COVID-19, la sociedad, el Estado, las empresas y las Instituciones de Educación Superior (IES) han tenido que replantearse la manera en que enfrentan las situaciones cambiantes del entorno. El sentido de una educación clásica o tradicional se ha visto rebasado ante la necesidad de una educación virtual, sincrónica, asincrónica y remota donde el uso de las Tecnologías de la Comunicación y la Información (TIC) sirve como puente para el desarrollo de una educación apegada a las necesidades de la actualidad. Los sistemas educativos se han topado con una serie de elementos externos que aquejan sus funciones sustantivas internas produciendo la necesidad de replantear sus esquemas funcionales. Derivado de ello, surge la duda sobre ¿cuáles son todos esos elementos que encierra el cambio hacia una educación virtual y digital?, y ¿hasta qué punto es este el futuro de la Educación Superior? En el entendido de que el futuro ha alcanzado al presente, o viceversa, la época actual ha dejado varias preguntas al aire y la necesidad de realizar reflexiones al respecto. Por tanto, el presente ensayo es un acercamiento al fenómeno de las necesidades de una educación superior futura desde una perspectiva del cambio institucional y la generación de utopías y distopías como mitos que proveen de certidumbre a las IES ante los retos que deben enfrentar. A través del texto se realizan diferentes reflexiones sobre las posibilidades que existen y la tarea de poner en el escenario a los actores involucrados, junto con las políticas para entrever las carencias y vicisitudes de una educación en tiempos posmodernos.
\end{abstract}

Palabras clave: mitos - cambio institucional - analfabetismo digital - utopías.

\begin{abstract}
Faced with the problems derived from the COVID-19 pandemic, society, the State, companies, and Higher Education Institutions (HEIs) have had to rethink the way they deal with changing environmental situations. The meaning of a classical or traditional education has been overtaken by the need for a virtual, synchronous, asynchronous and remote education where the use of Communication and Information
\end{abstract}

* Doctor en Gestión de la Educación Superior. Líneas de investigación: Cambio institucional, políticas educativas, gestión institucional. Instituto Tecnológico José Mario Molina Pasquel y Henríquez Campus Zapopan. México. guillermo.gonzalez@zapopan.tecmm.edu.mx 
Technologies (ICT) serves as a bridge for the development of an education attuned to the needs of our times. Educational systems have run into a series of external elements that affect their substantive internal functions, leading them to rethink their functional schemes. Thus, the question arises as to which are the elements that a change towards a virtual and digital education entails, and to what extent this is the future of Higher Education. Insofar as the future has reached the present, or vice versa, the times we live in have raised several questions in the air as well as the need to reflect on them. This essay is an approach to the phenomenon of the needs of a future higher education from the perspective of institutional change and the generation of utopias and dystopias as myths that provide certainty to HEls in the face of the challenges they must confront. We reflect on the possibilities available to us and the task of deploying the actors and the policies required to observe the shortcomings and vicissitudes of an education in postmodern times.

Keywords: myths - Institutional change - digital illiteracy - utopias.

\section{Introducción}

Desde tiempos remotos, los mitos y utopías han acompañado a las sociedades como parte central de la comprensión de lo cotidiano, lo inexplicable e incomprensible. Los mitos entonces surgen como elementos de asimilación de la realidad, acompañando con ello la relación generada entre la ilusión y lo concreto (Eliade, 1992). De igual forma, el mito toma cabida en el simbolismo entre la confrontación de la realidad con lo imaginario, ya que involucra una disputa de pensamientos que adecuan, según sus necesidades, el entorno donde se crea para darle un sentido, quizá más práctico, quizá más idealizado, de la situación vivida. Alrededor de esto circundan espacios de convivencia donde los individuos confrontan realidades apegadas a la racionalidad como medio de asimilación de su entorno, que a su vez les permiten establecer parámetros de asimilación con los cuales adaptarse a los cambios que surgen (Elster, 2010). Asimismo, los elementos que le dan forma a las acciones humanas en sus interrelaciones con otros individuos llevan una fuerte carga apegada a las reglas, normas y rutinas institucionalizadas en cada uno de los espacios de convivencia, que sirven como elementos para la adaptación a las nuevas circunstancias del entorno (Castoriadis, 2013).

El recuento con el cual se caracteriza a las sociedades desde tiempo remotos va de la mano con el proceso histórico, el sistema político y la trascripción cultural desarrollada en los entrecruces sociales (Wallerstein, 2016). Ahora bien, dentro de las concepciones de sociedades futuras o futuros sociales, existe una porción de irrealidad que permite dotar a los espacios sociales de una fuerte dosis de certidumbre ante la complejidad (Schutz, Luckman, 2001). En este punto es donde las utopías surgen como un mito que permite acentuar un presente plagado de un alto grado de irracionalidad. Sin embargo, ¿qué tanto esos mitos se convierten en parte integral

Diálo@os sobre Educación año 12 | número 22 | enero-junio 2021 | ISSN 2007-2171 
de un presente que no deja de gestarse? ¿hasta qué punto la realidad del mito se institucionaliza a grado tal de visualizarse como una solución ante un futuro no previsto? Los elementos principales de las preguntas anteriores llevan a la reflexión sobre los hilos que conducen y guían los procesos de cambio en los sistemas sociales. En ellos, se encuentran factores tales como las políticas, las estructuras, los sistemas de intercambio e incentivos, la toma de decisiones y la construcción de un imaginario social basado en una realidad desde donde se genera una visión respecto a los espacios en que se concretizan los cambios institucionales (March, Olsen, 1997).

Dentro de estos mitos y utopías, construidos como base de una aseveración hacia la modernidad, la vinculación existente entre sus actores y los medios con los cuales generan la comunicación, revelan la manera en que se cumple el objetivo del cambio (Meyer, Rowan, 1977). Ahora bien, cuando entran en función los elementos de un escenario global en que los actores se multiplican y diversifican, se aprecian entonces espacios imaginarios que traspasan la barrera del mito y se convierten en una realidad necesaria (Berger, Luckman, 1986). Si se puntualiza un poco este aspecto, se puede mencionar que los sistemas globales y modernos generan reglas de convivencia en espera de menguar la incertidumbre causada por la llegada de un futuro. Lo anterior permite la discusión sobre los elementos que contiene una época moderna, o posmoderna desde una perspectiva de los individuos y su contexto respecto a las situaciones concebidas como naturales (Lipovestky, 2000).

Como fue percibido por algunas mentes brillantes de mediados del siglo XX, el siglo XXI traería consigo una serie de elementos que complejizarían la convivencia, interacción y valoración humanas. Los escenarios previstos describen un futuro plagado de avances tecnológicos, supercomputadoras, inteligencia artificial, viajes al espacio, ciudades flotantes, pero a su vez, planteaban un mundo cada vez más caótico y desprovisto de humanidad. Los simbolismos encontrados en las intuiciones de estas grandes personalidades llevan hoy a la pregunta sobre la manera en que se enfrenta la realidad actualmente y cómo esa llamada posmodernidad altera las relaciones entre la sociedad, el Estado y las universidades de tal manera que genera incertidumbre respecto al grado de asimilación y adaptación a los cambios. Sin embargo, esta visualización del futuro apegado a un presente latente y constante ha llevado al análisis sobre la actualidad y el valor que tiene el cambio respecto a los procesos que se llevan a cabo para enfrentar las distintas problemáticas sociales.

Por ello, el presente ensayo toma como base las reflexiones sobre un escenario futuro en que se visualizan diversas posibilidades para recrear un presente repleto de incertidumbre. La relación directa que tienen los actores con sus espacios de interacción genera la posibilidad de imaginarse dentro de posibilidades en el tiempo que provean de un cierto grado de tranquilidad ante las diversidades del entorno. Se parte entonces de la perspectiva de tres visiones presentes que reúnen la situación que ha traído la pandemia respecto al desarrollo de competencias digitales, las problemáticas que aquejan a las universidades, los entornos globales, los 
procesos de cambio institucional, así como las perspectivas desde las cuales se puede imaginar un futuro utópico, distópico o retrópico en la educación superior.

\section{Las universidades desde un sistema mundo: mitos y utopías sobre la época posmoderna}

El escenario actual, sin despojarlo de su sentido complejo, ha generado la idea de que la modernidad nos ha sobrepasado. Pero, ¿por qué en un lapso de tiempo tan corto el contexto global se dio cuenta que existe la necesidad de un cambio en muchos de los aspectos sociales? ¿fue la pandemia de inicios del año 2020 la que permitió y exige un cambio hacia una perspectiva distinta? ¿son acaso los elementos tecnológicos los que se requieren para enfrentar una crisis de magnitud global? ¿qué pasará con aquellos individuos que no se adapten al cambio, ya sea por insurrección, imposibilidad o incapacidad? ¿son acaso las universidades fuentes latentes de cambio y deben adaptar sus procesos, actividades y sistemas hacia un espacio post-moderno, post-pandemia, post-crisis social?

Además de los sistemas económicos, uno de los espacios que más repercusiones ha tenido al enfrentarse a la pandemia son las universidades. A través del tiempo, los cambios realizados en los sistemas educativos en el contexto mexicano han sido el símbolo de reformas y políticas deterministas apegadas a un entorno global (Kent, 2016). Actualmente, la influencia que generan los esquemas globales permea el ámbito de las IES a grado tal que el mismo sistema se supedita en muchos de sus elementos a esos agentes de exigencia externos. Por ello, las políticas tanto de nivel global como regional, han sido un punto de inflexión para las universidades ya que producen, o por lo menos intentan, cambios internos en busca de una adaptación y adopción de las reglas con las cuales se considera deben jugar (Powell, DiMaggio, 1999). Se nota entonces un ámbito de interacción constante donde los cambios en el medio ambiente producen una adopción constante de nuevas reglas, normas y rutinas que los individuos asumen como básica y necesaria (Rowan, Meyer, 1977).

En este futuro alcanzado a la fecha, las IES han enfrentado diversas vicisitudes desde la década de los ochenta, cuando los procesos de reforma desencadenaron cambio en el sistema, la estructura y las relaciones existentes entre los actores (Acosta, 2013). Durante esa etapa llamada de "modernización" se avizoraba un futuro donde las universidades cumplieran con indicadores de calidad, evaluación y acreditación con un sentido de competitividad y con las cuales modificaban sus procesos y entornos metodológicos (Grediaga-Kuri, 2011). Siguiendo la línea de la actualidad respecto a la manera en que las instituciones educativas y sus actores enfrenan los procesos de adaptación a un esquema digital, tecnológico y virtual, las universidades ahora deben adoptar aquellos mecanismos que les permitan establecer líneas de acción para que los espacios pedagógicos, administrativos, políticos y culturales sean reformulados y se apeguen al constante ritmo de cambio social (Rama, 2018).

Diólo@os

sobre Educación año 12 | número 22 | enero-junio 2021 | ISSN 2007-2171 
Dentro de los espacios de modernización institucional, las universidades actualmente enfrentan al reto de confrontar la problemática mundial mediante una visión distinta, centrada en modificar los estilos, recursos y escenarios de la forma en que se generan las estrategias pedagógicas, las intenciones didácticas, la evaluación y los medios de impartir la educación (Cady, Olson, Shea, Grenier, 2018). Dadas las necesidades actuales, la virtualización toma un protagonismo imperante, sin embargo, no es un elemento nuevo, sino que es requerimiento que hace tiempo se trabaja, pero que no tenía el interés, ni la necesidad latente generada por el surgimiento de la pandemia. Dada la experiencia vivida en los primeros meses del año 2020, los recursos didácticos en los entornos educativos fueron múltiples y variados, al igual que las problemáticas, por lo que nos encontramos con diversos y distintos escenarios creados por las instancias públicas, privadas y sociales, para modificar sus esquemas en la impartición de educación. Lo anterior llevó a que los objetos y sujetos de aprendizaje cambiaran de una modalidad tradicional a una virtual de una forma drástica e intempestiva.

No obstante, en el cambio por necesidad se nota un rezago muy significativo en muchos de los elementos concernientes a la misma filosofía de la educación y a los mecanismos pedagógicos que se requieren para un modelo de educación distinto al tradicional (Rama, 2018). Antes de prever las posibilidades de estrategias a seguir, es necesario realizar un recuento de aspectos importantes para el modelaje organizacional y pedagógico que sirvan de base para la modificación de los métodos clásicos de impartir educación. Entre estos se encuentran las capacidades del Estado, así como de las IES, para proveer los servicios educativos que logren cumplir con objetivos de las profesiones. Se puede inferir que existen un par de variables que afectan directamente el tránsito hacia una educación virtual, y que van desde las asimetrías ${ }^{1}$ (Marúm, 2011) y las desproporciones, ${ }^{2}$ tanto en la calidad educativa como en el acceso a los medios necesarios para obtenerla y que sea un referente de una educación para la vida (Fernández, 2010).

En este tenor, el acercamiento a una educación virtual conlleva varios elementos inmersos en la currícula y los programas educativos, que muchas veces no son atendidos de manera adecuada, ya sea por falta de práctica o de herramientas para su ejecución (Leckey, McGuigan, 1997). La problemática que trae el mudar de un tipo de educación tradicional a uno regido por ambientes virtuales, conlleva el diseño de una estructura bien definida y prestablecida desde el ámbito institucional, que a su vez complejiza las relaciones internas en las IES, ya que esto involucra que, tanto docentes como alumnos modifiquen sus rutinas y las apeguen a nuevas reglas y procesos (García, 2011). Ahora bien, la conformación de ambientes virtuales involucra la exis-

1 Para explicar las asimetrías educativas se toma la analogía de Wallerstein (2016), que hace alusión a los procesos sociales donde hay diferencias entre capacidades institucionales para responder a las problemáticas, así como de los docentes y alumnos dentro de los procesos de enseñanza-aprendizaje.

2 El concepto de desproporción se retoma de Wallerstein (2016), para concebir la manera en que los sistemas educativos tratan de ser inclusivos e integradores, pero se topan con barreras sociales, económicas, políticas y personales que no permiten que la educación llegue de la misma manera a aquellos que la demandan. 
tencia de una serie de elementos tales como: a) e-learning (multimedios); $b$ ) on-line-learning (ambiente hipertextual); c) m-learning (sistemas móviles), que permitan abastecer las necesidades y requerimientos de una educación de calidad a los diversos sectores sociales (Rodríguez, Meseguer, 2018).

Aunado a lo anterior, la generación de los ambientes virtuales con los cuales hacer uso de estos elementos estratégicos, requiere un modelo de aprendizaje que entrelace la formación, la relación y la interacción de los aprendizajes en docentes, alumnos y directivos, así como los recursos tecnológicos necesarios para su implementación en las clases (Rodríguez, Chávez, 2020). Para el logro significativo de los mismos, se deben ligar conceptos tales como aprendizaje social, aprendizaje significativo, aprendizaje colaborativo, aprendizaje cooperativo y el aprendizaje autónomo, como base de una alternativa que acerque más los procesos de enseñanzaaprendizaje a los ambientes creados por las y los docentes (Guillén, 2017). Si esto se consigue, puede entonces pensarse en la generación de competencias digitales tanto en docentes como en el alumnado desde una perspectiva de integración y aprovechamiento de los recursos que abarque el conocimiento, las habilidades y la actitud generada en relación con las TIC y su contexto de aplicación (Silva et al., 2018).

En el plano mencionado en el párrafo anterior, las competencias docentes son indispensables ya que con ellas se establece la relación didáctica y la forma en que se generan las estrategias (Pérez, Mena, Pereida, 2019). La práctica pedagógica cotidiana en el plano educativo, ya sea en la modalidad presencial o virtual, involucra que las y los docentes desarrollen habilidades enfocadas a las competencias pedagógicas, sociales y técnicas como base de una integración de los planos pedagógicos al ambiente recreado en el aula (Araujo, 2019).

En este contexto, la educación en ambientes virtuales debe contener ciertas características que permitan su correcta implementación en ambos casos (virtual y presencial), ya que muchas veces difiere en cuanto a particularidades técnicas y recursos, tanto financieros como de aprendizaje, que obstaculizan la labor e interacción en clase (Coll, Mauri, Onrubia, 2008). Cuando alguno de los aspectos anteriores falla, la transición tiene dificultades pues los procesos no se concretan, ya sea por falta de visión política o por la ausencia de una estrategia que garantice la calidad en su implementación (De Laat et al., 2007). Otro de los elementos que también tiene una gran repercusión es el entorno económico, social y cultural del lugar donde se quiera realizar pues ello simboliza la adecuación, o no, a un ambiente distinto del acostumbrado (Facundo, 2004).

Sin embargo, se encuentra aquí otro punto de controversia referente a la alfabetización digital que se tiene en los diferentes segmentos poblacionales y cuyo objetivo es proveer las competencias necesarias para el uso adecuado de los recursos virtuales y digitales (Sinisterra, 2005). El analfabetismo digital se puede considerar como una problemática latente producto de las desigualdades, desinformación o falta de capacitación, según sea el caso (Guillén, Ascen- 
cio, Tarango, 2016). En el caso de nuestro país, cerca de $40 \%$ de las personas no realizan o no tienen idea alguna de la manera en que los medios digitales pueden utilizarse. Esta problemática se acerca mucho a la descripción del fenómeno de la entropía ${ }^{3}$ como una analogía planteada en un medio en que la ausencia o carencia de medios obstaculiza el desempeño del cambio en elementos sociales, generando un alto grado de incertidumbre. Se puede aseverar entonces que, tanto la falta de competencias docentes digitales como la brecha que se crea por las asimetrías y desproporciones en los estudiantes, produce un vacío en los procesos de cambio que se quieren alcanzar (Zimmerman, 2001).

En este sentido, el analfabetismo digital genera factores determinantes para que el sistema educativo pueda evolucionar, o no, hacia una perspectiva virtual ya que frena en gran medida el desarrollo conceptual y empírico de su aplicación (Czernik, 2006). Lo anterior involucra que en cada uno de los lugares donde se pretenda generar un cambio deben existir los medios adecuados, así como de las capacidades personales e institucionales para utilizarlos, en la búsqueda de un desarrollo tecnológico aplicado a un entorno específico (Rodríguez, 2008). Cuando esta conjunción se realiza con el acompañamiento de una estructura normativa y política, el modelo de educación virtual, o que se apoye en la virtualidad, tiene mayores posibilidades de progresar, compensando con ello las brechas existentes entre sectores de la población y los mismos involucrados en la enseñanza-aprendizaje (Facundo, 2004).

Como contraste a este aspecto, las políticas y programas creadas por las universidades en conjunto con el Estado, deben responder a los ambientes externos e internos con estrategias que permitan el logro de la literacidad digital ${ }^{4}$ en docentes y alumnos (Aguilar, Ramírez, López, 2014). El concepto de literacidad se comprende, desde una perspectiva de la virtualidad, como aquel que combate el analfabetismo mediante mecanismos estructurales y simbólicos que facilitan el cambio a una modalidad en línea (Vargas, 2015). En este aspecto, el ideal sugerido en los espacios internacionales se direcciona hacia la adquisición y uso de medios digitales accesibles a la mayor parte de la población, que representen una mayor posibilidad de disminuir las brechas y desigualdades producidas por los mismos medios virtuales (UNESCO, 2011). Sin embargo, es bien sabido que no todas las personas tienen acceso directo a un computador, internet o alguno de los otros recursos digitales que les permitan adentrarse en un ambiente de educación digital, lo que provoca la existencia de márgenes amplios de desigualdades, así como disparidades socioeducativas (García, Aquino, Ramírez, 2016).

Ahora bien, tanto el analfabetismo digital como la literacidad digital, involucran un cambio significativo, no solo de las condiciones institucionales o de la capacidad adquisitiva para la

3 Para el presente trabajo se hace una analogía del término entropía desde una perspectiva de informática, apegado a la incertidumbre causada por la falta de información respecto a la capacidad que tienen los individuos para asimilar la información recibida y hacer uso de ella.

4 El término literacidad digital se refiere al acercamiento de los individuos hacia los ambientes digitales con el fin de hacer uso de ellos de manera tal que se comprenda como un acercamiento a la alfabetización; se logra sobre los espacios virtuales, la búsqueda de información y la comprensión de términos para su uso y aplicación.

Diálopos sobre Educación 
obtención de un aparato tecnológico, sino también en las rutinas, costumbres y la estructura generada en los espacios sociales (Czernik, 2006). En el entendido de que existe ahora una nueva normalidad respecto a una metáfora en que el mito del futuro llegó de pronto cual utópico o distópico ${ }^{5}$ acercamiento a una realidad que no se había atendido del todo. La relación o correlación existente entre las diferentes vertientes de la realidad que aquejó durante el año 2020 al sistema socioeconómico global, dejó una huella muy marcada respecto a la manera en que la interacción entre los individuos se dará de hoy en adelante. Con lo anterior se refuerza el sentido de lo clásico, lo actual, lo moderno y lo posmoderno desde un sentido de las posibilidades para imaginar un mundo distinto.

En el siguiente apartado se describe la situación a la que se enfrentan las IES como base de la interacción con los medios externos, su propia realidad, el futuro que se espera de ellas, y la posibilidad de recrearse como una instancia que permita disminuir las brechas sociales y digitales. Sin embargo, ante esta panorámica de la normalidad, las universidades y demás instituciones educativas se han topado con muchas problemáticas, no solo internas sino también externas, que han generado mucha incertidumbre respecto a las acciones a seguir a partir de este momento y en las subsiguientes fases del regreso a una normalidad.

La tarea que tienen ahora este tipo de instituciones conlleva el mirar a su alrededor para quizá fusionarse con él, crear su propio futuro, someterse a las reglas coercitivas de un mundo global que exige una adaptación a las nuevas normas, rutinas y costumbres como parte de la legitimización para establecer las bases de un compromiso general ante los retos por venir. A lo anterior puede uno preguntarse en este momento, ¿cómo respondieron las universidades ante el reto del cambio y la adaptación? ¿qué tanta influencia se generó en ellas al momento de estructurar un nuevo sistema ya antes visto y utilizado, pero no aplicado en su gran mayoría con un sentido estricto de la palabra? ¿hasta qué punto las y los docentes tienen las competencias antes mencionadas para adoptar un nuevo modelo que les confiere un grado alto de cambio? $Y$, sobre todo, ¿qué tanta incertidumbre produce el que el cambio confiera la posibilidad de un nuevo futuro con otras capacidades, otras competencias y otra forma de impartir la educación?

\section{El contexto de la normalidad en la posmodernidad: distopía, retrotopía y entropía entre los espacios sociales, políticos y las universidades}

A través del tiempo, la concepción de la realidad ha afectado de forma significativa el contexto donde se desarrollan las diversas situaciones de interacción humana. La percepción sobre el lugar donde interactuamos radica en la forma en que se crean las redes de convivencia en cada uno de los espacios. En ellos, se integran factores tales como economía, la sociedad, la cultura,

5 El término distopía, para el presente trabajo, es aquel que visualiza un futuro no deseable que puede generar problemáticas diversas en los diferentes espacios sociales, sea por la dificultad para proporcionar oportunidades para la mayoría o por las disrupciones que pueden desencadenarse en la conclusión de los objetivos que se buscan.

Diálo pos 
la biodiversidad, y en gran medida a lo largo de los siglos XX y XXI, la tecnología. Como se señaló en el apartado anterior, la dinámica actual conlleva el entrevernos en un espacio distinto de convivencia e interacción al cual la adaptación lograda permita obtener una visión sobre un mundo posible.

Al pasar por una etapa de cambio, tanto en la percepción como en la forma de convivencia, la sociedad, en conjunto con el Estado y las universidades, en primer lugar, tratan de comprender una realidad prevista pero no planeada, y adaptarse a una nueva forma de interacción, y en segundo, de entrever las diversas posibilidades de escenarios a los cuales enfrentarse (Powell, DiMaggio, 1999). Ahora bien, al ser tan complejo el contexto de las universidades, requiere una revisión minuciosa sobre la interrelación que existe en su interior y que a la vez vaya asociada a la capacidad institucional que se tenga para ejercer influencia en su entorno (Mejía, 2014). Aunado a ello, todas las manifestaciones sociales, políticas y económicas que conllevan un espacio moderno de cualquier tipo tienen por añadidura una dosis de simbolismo recreado por el espacio mismo donde se desarrolla.

Se encuentra entonces con una perspectiva en que, ante las problemáticas de pasar de un presente resguardado en un pasado, hacia un futuro que se apegue al presente y donde las posibilidades de adaptación permitan reducir la incertidumbre sentida, las IES deben de establecer estrategias clave que les permitan adaptarse a los cambios del entorno y ser un referente de apoyo social (Bauman, 2017). Al tratar entonces de conseguir un cambio, se debe de tomar en consideración aquellos elementos que estén presentes en el funcionamiento social y que tengan relación directa con el contexto donde las IES van a desempeñarse (Kent, 2016). En este aspecto es donde se interrelacionan los espacios de interacción estatales con las dinámicas institucionales de las universidades, para conducirlas hacia una adecuación del ambiente interno hacia las exigencias externas en un sentido de correlación elemental (Ansell, 2008).

Como ya es sabido, la modernización de la educación superior comenzada en la década de los ochenta ha influenciado en gran medida la manera en que las universidades a nivel global se adaptaron a un esquema mercantilizado (Wilkins, 2019). En esos años, el futuro de las universidades estaba marcado con una etiqueta de modernización que visualizaba un futuro distinto para la educación terciaria más apegado a reglas y normas de competencia, y que también era visto como una necesidad para que las universidades mudaran sus procesos, actividades y dinámicas hacia otras que sobrepasaran su pasado y presente (López Segrera, 2016). A la entrada del siglo XXI, las modificaciones en los espacios sociales, económicos, culturales y sobre todo tecnológicos, acercaban aún más ese mundo utópico con sus dotes de distópico que generaba disputas sobre el futuro de la educación. Para esclarecer este punto, es necesario decir que el sentido de utopía en el espacio de las IES puede considerarse como aquel en que las políticas y los cambios sugeridos provean a las universidades de una formación ideal respecto a lo que se considera deben tener. 
Tomando el sentido del término anterior, las IES ideales deben estar apegadas a las normas, políticas y sugerencias realizadas por las instancias internacionales para amoldar sus procesos, adaptar sus medios como parte de una necesidad de cambio y adoptar procesos alternos de calidad como clave para obtener la legitimidad (Popadiuk, Rivera, Bataglia, 2014). Sin embargo, en la época de la modernización, el futuro se veía como algo que aún le distaba algún tiempo para alcanzar la realidad presente, sobre todo en los espacios universitarios que suelen ser renuentes al cambio. Fue entonces que las TIC tomaron auge al pasar de ser solo un recurso didáctico, pedagógico y procedimental, a una necesidad latente en la educación (Constantino, Álvarez, 2010). A partir de esos años, los sistemas educativos comenzaron a adoptar modelos que fueran acorde a los requerimientos de un cada vez más exigente mercado de competencia global donde las competencias digitales y la comercialización de los servicios educativos provocaban un nuevo esquema en las universidades (Hernández, 2017).

Se genera entonces una disrupción entre la llamada educación tradicional y la educación virtual y/o a distancia. Los retos que enfrentan las universidades y los sistemas estales se encaminan al desarrollo de un plan que integrara una modalidad digital al subsistema de educación tradicional (García, Ruiz, 2020). Este tipo de transición o adaptación, implica que se tomen en consideración muchos elementos del entorno que permitan definir el alcance que se quiera tener y los medios con los cuales se cuenta para lograrlo (Santos, Mella, Sotelino, 2020). Sin embargo, también es necesario adherir los temas antes descritos sobre la alfabetización digital y la literacidad requerida e indispensable para que la virtualidad pueda servir como base en un sistema de educación incluyente (Saldivia, 2020).

Considerando que existen diferencias económicas y culturales en los diferentes contextos, las sociedades tratan de compensarlas con adecuaciones a sus sistemas de convivencia y organización. La manera en que estos sistemas se acoplan a una formación adaptable y moldeable, genera que los medios de producción y comunicación subsistan como una manera de sobrevivir en los espacios sociales (Elster, 2010). Ahora bien, la relación entre las IES y el esquema social ha ido modificándose en cierta forma conforme las propias necesidades así lo pidan. Referente a ello, el proceso que se buscaba de complementación educativa con el uso de las TIC en el aula y la formulación de un modelo dual, virtual o mixto, era utilizado en muchas de ellas para complementar sus funciones (Bringle, Clayton, 2020).

Sin embargo, a pesar de contar con los elementos anteriores, existen situaciones que salen completamente del contexto de asimilación creando rupturas en la línea general desde donde se desenvuelven las situaciones cotidianas. En este caso, se puede hacer alusión a un sentido de entropía que le quita el orden a la normalidad y a la concepción de una posibilidad de futuro acercando una circunstancia $X$ a un momento $Y$, creando con ello la necesidad de cambio. Pero, ¿en qué forma la adaptación a los cambios es parte de las circunstancias en el tiempo y en el espacio de la educación superior? Como bien es sabido, el año 2020 ha marcado drástica- 
mente el sentido de normalidad al que el presente y la realidad le daban una dosis de seguridad y certidumbre. Conforme se da la evolución del cambio producido surgen más preguntas sobre lo que nos depara el incomprendido futuro que se siente tan presente y que de ahora en adelante marcará una pauta, quizás, en los espacios educativos.

Si bien es cierto que cada IES en México cuenta con capacidades distintas y con modelos específicos con los que tratan de prestar educación, también lo es que su grado de avance y modernización tiene una influencia significativa en las personas que las conforman (Temple, 2014). Los sistemas políticos en el interior de las universidades forman redes de influencia desde las cuales se toman las decisiones y se realizan acuerdos para que exista un posicionamiento institucional (López et al., 2009). Esto surge como un ritual institucional que es de llamar la atención pues mientras se atienden temas políticos, se pueden desatender temas académicos y administrativos que son clave para la supervivencia institucional (Gómez, 2014). ¿A qué va el asunto anterior? Al tema de la previsión de escenarios futuros posibles y la planeación de estrategias para sobrellevar las acciones necesarias para enfrentarlos. Es precisamente en este punto donde las distopías, como aquellos espacios de un futuro no deseado, pueden afectar a las universidades al modificar sus escenarios hacia esquemas homologados que le restan autonomía y capacidad de diferenciación.

En este acercamiento al sentido interno de las IES, donde los grupos de poder compiten por asegurar una posición, se generan grandes diferencias entre la apertura docente respecto a la adopción y adaptación, o no, de nuevos esquemas institucionales (Brady et al., 2014). La influencia interna que lleva el proceso educativo, muchas veces recaen totalmente en las y los docentes, alejando a la institución de una de sus responsabilidades: la formación y capacitación docente. Si a esto se le agrega la influencia de factores normativos externos, del mercado laboral y de las exigencias sociales, las universidades se ven inmersas en un caleidoscopio de posibilidades que complejizan la toma de decisiones (Aboites, 2010). Es ahí cuando deben de enfrentar los retos de forma conjunta para asegurar que respondan de forma adecuada, tanto con la impartición de educación como con la generación de proyectos que fomenten la participación ciudadana, a las necesidades, carencias y problemáticas del entorno donde se desenvuelven (Dutrénit, De Fuentes, Torres, 2010).

Para dar una pequeña idea de lo que se trata de abordar en el contexto actual, en México existen 3,762 IES y 6,359 centros de trabajo repartidos a lo largo de los 32 estados de la república, que atienden a un total de 4'561,792 alumnos(as) distribuidos de la siguiente manera: 2'954,222 en el sector público, de los cuales $91.75 \%$ lo realiza en modalidad escolarizada, mientras que el restante $8.25 \%$ lo hace en modalidad no escolarizada. Por su parte, en el sector privado estudian 1 '607,570, entre ellos, $71.84 \%$ en modalidad presencial, mientras que el restante $28.16 \%$ se encuentra en la modalidad semiescolarizada. Dicho número de estudiantes se encuentra dentro de alguno de los 28,151 programas de licenciatura y los 11,209 de posgrado que se ofrecen en el total de las instituciones (DGESU, 2020). 
La magnitud de un sistema educativo terciario como el de México involucra que los planes que se generen contengan una fundamentación clara de los aspectos axiológicos, normativos, académicos y de gestión, respecto a las líneas que se piensa establecer y los medios necesarios para alcanzarlas (Vértiz, 2011). También se requiere un estudio que permita establecer diferencias institucionales en los 13 subsectores educativos que existen, para describir los escenarios que se pintan en cada uno de ellos, logrando con ello tener una perspectiva más centrada en las necesidades particulares (Kent, 2016). Ahora bien, no solo es indispensable el estudio que demarque las diferencias sino también las circunstancias que afectan a las universidades para enfrentar las situaciones presentes y futuras. El entorno de contingencia permitió visualizar muchas problemáticas internas y externas que tienen las universidades, pero también puso acento en la situaciones y diferencias que existen entre los docentes, estudiantes, los sectores económicos y las brechas entre algunas personas respecto a sus competencias digitales.

Para tratar de esclarecer un poco al panorama educativo actual, la Asociación Mexicana de Universidades e Instituciones de Educación Superior (ANUIES), en conjunto con la Secretaría de Educación Pública (SEP), realizaron un plan de acción basado en un estudio diagnóstico de las IES con la intención de enfrentar el periodo de contingencia. En él, se plasman diversos elementos concernientes a los problemas que los aquejan en este periodo de confinamiento con el propósito de que surjan estrategias específicas para mantener los servicios educativos y contribuir a la salud y el bienestar social. El diagnóstico se realizó en 582 IES en todo el país donde cada una de ellas plantea sus diversas problemáticas, así como la manera en que las enfrentaron. Uno de los puntos centrales de lo anterior, es el rescate de aquellas y aquellos estudiantes que se encuentran en situaciones de desigualdad pues $33 \%$ de las IES realizaron acciones para atender a estudiantes con desventajas económicas (SEP, ANUIES, 2020).

Del mismo modo, se señala que $26 \%$ de las instituciones educativas realizaron acciones para apoyar a las diversas comunidades y a la sociedad en general, mediante la fabricación de gel antibacterial, estudios científicos sobre el virus, adecuación de laboratorios para realizar diagnósticos, asesoría sobre salud emocional, entre otros. Al ver la magnitud de lo que había por afrontar, algunas de las IES decidieron tomar iniciativas que fueran acordes a las necesidades sociales en cuestiones de salud, mediante las cuales ayudar a solventar las deficiencias y carencias existentes en el sector. Aunado a esto, en las diferentes instancias, tanto públicas como privadas, se impulsaron iniciativas para el fomento de proyectos que apoyaran a los diversos sectores que se vieron afectados con la pandemia.

Por su parte, desde el aspecto educativo, ante la premura y necesidad de mudar a una modalidad en línea, $14 \%$ de las universidades señalaron que elaboraron manuales, procesos y/o material de capacitación para docentes y alumnos que permitieran pasar a esta modalidad y no interrumpir las labores académicas. Las dificultades generadas por la pandemia produjeron 
que las diversas instancias educativas tomaran decisiones respecto a la manera de enfrentar la situación, dando como resultado una serie de elementos estructurales y académicos que permearon los distintos escenarios educativos (SEP, ANUIES, 2020).

Ante esta situación tan inesperada, y como una parte elemental del proceso de adaptación y cambio, las IES deben tomar una serie de consideraciones que les permitan la realización de un análisis situacional que logre un panorama más certero respecto a las circunstancias producidas. Dentro de ellas se destacan: a) factores pedagógicos respecto a la manera de fomentar la praxis en ambientes virtuales, $b$ ) desarrollar estructuras curriculares que abarquen las necesidades psicopedagógicas de los programas, c) el acompañamiento de sistemas epistemológicos con los cuales sustentar el conocimiento generado y su aplicación, y d) la formación de espacios administrativos que fomenten el desarrollo de una base de servicios educativos en una perspectiva ya sea a distancia, semipresencial o híbrida (Rama, 2019). De ello se puede desprender una serie de cualidades específicas que Preradovic y Stark (2019) señalan como básicas para realizar un modelo pedagógico integral para el impulso al cambio en los ambientes virtuales y que se puede apreciar en el siguiente cuadro:

Cuadro 2. Cualidades estándar para el servicio de aprendizaje

\begin{tabular}{|l|l|l|}
\hline Cualidades & Características & Necesidades \\
\hline $\begin{array}{l}\text { Relevancia y significado para } \\
\text { comunidades y estudiantes. }\end{array}$ & $\begin{array}{l}\text { Adecuación de programas educa- } \\
\text { tivos, competencias adquiridas, } \\
\text { infraestructura física y virtual. }\end{array}$ & $\begin{array}{l}\text { Programas de capacitación, } \\
\text { Plataformas virtuales, } \\
\text { Alfabetización digital, } \\
\text { Hibridación curricular. }\end{array}$ \\
\hline $\begin{array}{l}\text { Valoración de las organizaciones } \\
\text { comunitarias, públicas y empre- } \\
\text { sariales. }\end{array}$ & $\begin{array}{l}\text { Sincronización de las problemá- } \\
\text { ticas comunitarias, públicas y } \\
\text { empresariales acorde a las posibi- } \\
\text { lidades virtuales. }\end{array}$ & $\begin{array}{l}\text { Plan de integración y respuesta } \\
\text { ante problemáticas, } \\
\text { Diagnóstico situacional. }\end{array}$ \\
\hline $\begin{array}{l}\text { Reciprocidad en el intercambio } \\
\text { de información y beneficios. }\end{array}$ & $\begin{array}{l}\text { Generación de canales de co- } \\
\text { municación con los diferentes } \\
\text { actores. }\end{array}$ & $\begin{array}{l}\text { Plataforma informativa e interac- } \\
\text { tiva. }\end{array}$ \\
\hline $\begin{array}{l}\text { Objetivos compartidos, alcanza- } \\
\text { bles y evaluables. }\end{array}$ & $\begin{array}{l}\text { Generación de una visión conjun- } \\
\text { ta para sobrellevar la problemáti- } \\
\text { ca y generar opciones de apoyo. }\end{array}$ & $\begin{array}{l}\text { Plan de integración, } \\
\text { Políticas públicas e instituciona- } \\
\text { les, } \\
\text { Financiamiento, } \\
\text { Sensibilización. }\end{array}$ \\
\hline $\begin{array}{l}\text { Integración con los programas } \\
\text { académicos. }\end{array}$ & $\begin{array}{l}\text { Incorporación de los medios vir- } \\
\text { tuales a la currícula, estrategias y } \\
\text { formación académica. }\end{array}$ & $\begin{array}{l}\text { Hibridación curricular, } \\
\text { Programas de formación e inte- } \\
\text { gración docente. }\end{array}$ \\
\hline $\begin{array}{l}\text { Aprendizaje cívico y académico } \\
\text { cos y deontológicos en espacios } \\
\text { presenciales y virtuales. }\end{array}$ & $\begin{array}{l}\text { Formación ética, } \\
\text { Políticas de uso de datos e infor- } \\
\text { mación. }\end{array}$ \\
\hline
\end{tabular}




\begin{tabular}{|c|c|c|}
\hline $\begin{array}{l}\text { Ofrecer oportunidades de apren- } \\
\text { dizaje a las comunidades para } \\
\text { todos los involucrados. }\end{array}$ & $\begin{array}{l}\text { Expansión de la capacidad ins- } \\
\text { titucional en la impartición de } \\
\text { educación. }\end{array}$ & $\begin{array}{l}\text { Programas de alfabetización di- } \\
\text { gital, } \\
\text { Programas de aprendizaje para } \\
\text { la vida, } \\
\text { Políticas de integración. }\end{array}$ \\
\hline $\begin{array}{l}\text { Colaboración y participación } \\
\text { de estudiantes en proyectos de } \\
\text { apoyo. }\end{array}$ & $\begin{array}{l}\text { Impulso de proyectos de investi- } \\
\text { gación que coadyuven a la resolu- } \\
\text { ción de problemáticas. }\end{array}$ & $\begin{array}{l}\text { Bancos de proyectos institucio- } \\
\text { nales, } \\
\text { Políticas institucionales, } \\
\text { Planes de emergencia e integra- } \\
\text { ción. }\end{array}$ \\
\hline $\begin{array}{l}\text { Reflexión guiada que impulse y } \\
\text { vincule la experiencia con el tema } \\
\text { y la exploración de múltiples } \\
\text { perspectivas. }\end{array}$ & $\begin{array}{l}\text { Integración de medios virtuales y } \\
\text { digitales para el apoyo y colabo- } \\
\text { ración de las comunidades acadé- } \\
\text { micas en el entorno. }\end{array}$ & $\begin{array}{l}\text { Plataforma virtual, } \\
\text { Canales de comunicación, } \\
\text { Capacitación docente y admi- } \\
\text { nistrativa sobre metodologías } \\
\text { divergentes. }\end{array}$ \\
\hline $\begin{array}{l}\text { Apoyo estudiantil por parte de la } \\
\text { institución. }\end{array}$ & $\begin{array}{l}\text { Requerimientos para la facili- } \\
\text { tación de las y los estudiantes } \\
\text { con problemáticas diversas para } \\
\text { adentrarse en medios virtuales y } \\
\text { tecnológicos. }\end{array}$ & $\begin{array}{l}\text { Mecanismos de acción institucio- } \\
\text { nal. } \\
\text { Gestión institucional, } \\
\text { Estrategias de acción. }\end{array}$ \\
\hline Tiempo suficiente & $\begin{array}{l}\text { Adecuación de los programas, } \\
\text { asignaturas y medios a las ne- } \\
\text { cesidades latentes de las y los } \\
\text { estudiantes, docentes y adminis- } \\
\text { trativos. }\end{array}$ & $\begin{array}{l}\text { Programación acorde a necesi- } \\
\text { dades, } \\
\text { Hibridación educativa, } \\
\text { Cambio de paradigma presencial } \\
\text { a digital. }\end{array}$ \\
\hline Medios de evaluación & $\begin{array}{l}\text { Adecuación de metodologías de } \\
\text { evaluación a los requerimientos y } \\
\text { necesidades para la formación de } \\
\text { competencias. }\end{array}$ & $\begin{array}{l}\text { Unificación de rubricas, } \\
\text { Metadatos para la toma de deci- } \\
\text { siones, } \\
\text { Planes de seguimiento. }\end{array}$ \\
\hline $\begin{array}{l}\text { Diseño de un impacto continuo y } \\
\text { sostenible }\end{array}$ & $\begin{array}{l}\text { Generación de una visión sobre el } \\
\text { futuro educativo y la integración } \\
\text { de los medios virtuales y tecnoló- } \\
\text { gicos como parte importante de } \\
\text { la formación. }\end{array}$ & $\begin{array}{l}\text { Plan estratégico, } \\
\text { Políticas institucionales, } \\
\text { Integración curricular. }\end{array}$ \\
\hline Transdisciplinariedad & $\begin{array}{l}\text { Formación con base en diversas } \\
\text { posibilidades que integren en la } \\
\text { educación a distancia y presencial } \\
\text { elementos que la nutran e impul- } \\
\text { sen. }\end{array}$ & $\begin{array}{l}\text { Plan estratégico, } \\
\text { Políticas públicas e instituciona- } \\
\text { les, } \\
\text { Financiamiento, } \\
\text { Generación de redes académicas. }\end{array}$ \\
\hline
\end{tabular}

Fuente: elaboración propia con base en Preradovic y Stark (2019).

Los elementos antes señalados dan pie a la propuesta sobre una integración estratégica de elementos que apoyen no solo al apartado institucional de las universidades, sino al conjunto de actores presentes en el sistema educativo. De lo anterior se puede inferir que la necesidad de 
repensar el sentido institucional en un espacio institucionalizado ${ }^{6}$ puede apoyar el proceso de cambio visualizándolo como algo necesario, incluso en un escenario emergente como el que se vive con el periodo de pandemia COVID-19 (Meyer, Rowan, 1977). Por su parte, para que exista una modificación sustantiva en los esquemas tradicionales, es necesario que se conjuguen elementos internos y externos como parte de una asimilación sostenida y necesaria de lo que se generará en los espacios institucionales y personales de las y los involucrados (González, 2017). Por tal motivo, los ambientes virtuales requieren una atención especial al momento de tratar de identificar los objetivos y metas que se quieran alcanzar y no solo como un factor para solventar una eventualidad o como paliativo de la carencia de infraestructura, planeación y políticas emergentes (Bringle, Clayton, 2020).

\section{El enfrentarse a la realidad: aspectos referentes al cambio institucional en el periodo de pandemia}

Es interesante marcar que en periodos de crisis y guerra, los esquemas institucionales tienden a modificarse para adaptarse a los requerimientos que el entorno plantea, en una lógica que responde a los señalamientos de sobrevivencia (March, 1994). Empero, en el trance del cambio surgen diversas problemáticas que deben ser atendidas, sobre todo si no se cuenta con los elementos indispensables para solventar las vicisitudes que conlleva el panorama a seguir bajo la incertidumbre generada. Al respecto, en este periodo de transe, las universidades, en conjunto con el sector público, deben solventar los inconvenientes que surjan mediante el desarrollo de una estructura académica estable que haga uso de las TIC y de todos aquellos recursos virtuales que apoyen el desarrollo de una plataforma estructural digital-virtual (Pérez, Mena, Pereida, 2019). Ante estos elementos, la ANUIES (2020) señala las siguientes problemáticas como las más asiduas que se detectaron en los entornos universitarios: 1 ) resistencia o falta de preparación para migrar a la educación a distancia; 2) problemas de comunicación a distancia con los equipos de trabajo; 3) poco tiempo para reaccionar, tomar decisiones y adecuar procesos; 4) incertidumbre sobre la duración de la contingencia; y 5) falta de información clara y oportuna de las autoridades, tanto federales como estatales, municipales e institucionales.

La información anterior sirve para reflexionar sobre las necesidades que las IES tienen en forma general en sus procesos y funciones, así como la manera en que enfrentan aquellos aspectos que requieren atención. De lo anterior nacen las problemáticas que los aquejan al momento de establecer la modalidad en línea, los espacios virtuales y/o las modificaciones en los planes y programas que afectan el mudar de una clase presencial a una virtual (Constantino, Álvarez, 2010). Muchas de las veces la falta y/o ausencia de una infraestructura institucional repercute en gran medida en el desempeño de una educación en ambientes virtuales. Si a esto se añade la

6 Entiéndase el sentido de institucionalizado como lo describe Lapassade (2008) desde el punto en que las acciones, rutinas, normas o mitos se vuelven parte de la realidad de los individuos, formando con ello un sentido de pertenencia y aceptación a los diversos espacios sociales. 
falta de políticas públicas, la poca o nula capacitación docente en ambientes virtuales y el analfabetismo por parte de la mayoría de los involucrados, se genera una maraña de elementos a seguir y con los cuales trabajar en la propuesta para la integración de un modelo adecuado a las necesidades del entorno (Serra, Fe, Roca-piera, 2020).

Derivado de la atención que se le prestó a la impartición de educación, la ANUIES señala que, hasta el 8 de junio de 2020, las IES reportaron la atención de $71 \%$ de los estudiantes mediante la modalidad a distancia. Sin embargo, existe aquí un punto al cual prestar atención ya que el alto porcentaje señalado no significa que esta misma se prestara de forma adecuada y cumpliera el objetivo de la materia o el desarrollo de competencias. A esto se puede agregar que, a pesar de que la información puede presentarse como un índice elevado de atención a estudiantes, $26 \%$ de los mismos indicaron no contar con una computadora o tableta y no tener acceso a internet de manera constante en casa (SEP, ANUIES, 2020). Cabe señalar que los datos anteriores no abarcan la totalidad de IES en el país, y el problema suele acrecentarse en aquellas comunidades donde los servicios digitales y de internet no son muy accesibles.

Para dar un panorama general del uso de los medios digitales y el internet, el Instituto Federal de Telecomunicaciones (IFT) indica que en México se encuentran 80.6 millones de usuarios de internet y 86.5 millones de personas que cuentan con celular. De ellos, $76.6 \%$ de usuarios se ubican en zonas urbanas, mientras que en las zonas rurales hacen uso de internet $47.7 \%$ de los habitantes. Para conectarse a la red, $95.3 \%$ lo hace por medio de teléfonos móviles, 33.2\% lo realizan por computadora portátil, mientras que $28.9 \%$ hacen uso de computadoras fijas. Lo anterior indica que el índice de capacidad para obtener un acceso a internet es alto, no tanto como se desearía, pero, a pesar de ello, es bajo el nivel de uso que se tiene de las computadoras portátiles. Aunado a ello, el uso que se hace por parte de los usuarios del internet va encaminado a que $91.5 \%$ lo utilizan como entretenimiento, $90.7 \%$ lo hacen para buscar información y 90.6\% para comunicarse con otras personas (IFT, 2020).

Si a los datos anteriores se le agrega el componente de las problemáticas que surgen en las bases de datos que generó el INEGI para 2019 respecto a la disponibilidad y el uso de las $\mathrm{TIC}$, se obtiene que $44.3 \%$ de los hogares poseen una computadora y de ellos, $44.6 \%$ lo utilizan como herramienta de apoyo escolar $y$, entre estos, $56.4 \%$ cuentan con acceso a internet como un recurso para realizar diversas actividades (INEGI, 2020). Por su parte, en el reporte realizado por la Organización para la Cooperación y el Desarrollo Económico (OCDE, 2020), México aparece como uno de los diez países con mayor crecimiento en la cobertura de banda ancha y penetración de la misma para el acceso a internet, pero ocupa el lugar número 82 en el mundo en cuanto a acceso a internet en escuelas. Es notable cómo los datos anteriores permean en el desarrollo de las competencias digitales pues $43 \%$ de las personas aún no tienen las habilidades y conocimientos necesarios para el uso de las tecnologías y los medios virtuales (INEGI, 2020).

Diólo@os 
Para tratar de solventar el periodo de contingencia de la pandemia, el consenso de las universidades reunidas en la ANUIES propuso la generación de diferentes recursos para lograr enfrentar la eventualidad. Entre las estrategias utilizadas por las IES resaltan el desarrollo de cursos de capacitación para la modalidad a distancia, la creación de herramientas de soporte técnico y asesoría en línea; la realización de acciones de capacitación adicional para el uso de las tecnologías; el diseño de materiales y guías; así como la creación de un número telefónico para la atención y soporte para asesorar a las y los docentes. De igual forma, las IES reportaron que 69\% de sus docentes están preparados para continuar labores de forma virtual, lo que indica que este porcentaje de docentes capacitados deben contar con las competencias, conocimientos, habilidades y destrezas requeridas para impartir cursos en la modalidad en línea (SEP, ANUIES, 2020).

Sin embargo, las competencias necesarias en el uso de los recursos para la impartición de clases en línea, establecen el desarrollo de habilidades en ámbitos como: 1) manejo de conceptos y funciones básicas de computadora; 2) Realiza tareas básicas de conectividad, instalación y seguridad del equipo de cómputo; 3) Maneja funciones básicas de los programas de productividad; 4) Muestra una actitud positiva para su actualización permanente en temas relacionados con las TIC; 5) Sabe cómo localizar y recuperar información; 6) Analiza y selecciona la información de manera eficiente; 7) Organiza la información recuperada de internet de manera adecuada; 8) Utiliza y presenta la información de manera eficaz, ética y legal (Rangel, 2015).

En este sentido, el uso de las competencias docentes digitales, debe tener un adecuado sustento de recursos tecnológicos que coadyuve al mejor funcionamiento de las sesiones y el logro de resultados en los procesos de aprendizaje (Salinas, 2004). Para contextualizar este punto, del total de instituciones que participaron en el plan de la ANUIES, destaca que 77 de ellas utilizaron una plataforma diseñada por ellas mismas para dar respuesta a las necesidades de la contingencia. Por su parte, 202 adquirieron una licencia de una plataforma comercial, mientras que 303 utilizaron herramientas digitales gratuitas de uso público (SEP, ANUIES, 2020). Si bien es cierto que los resultados indicados por las universidades es que poco a poco fueron afrontando los retos que traían la virtualidad y la digitalización, también lo es que aún no se tienen resultados palpables sobre el primer semestre de 2020 que permitan generar un diagnóstico de la situación y las líneas a seguir. De igual forma, tampoco se cuenta con datos precisos que indiquen el nivel de asimilación y adaptación que tienen, tanto docentes como alumnos, para enfrentar los medios virtuales y la brecha digital.

Existen entonces aspectos básicos que se han denotado en el sistema educativo terciario por medio de la pandemia y que pueden resumirse en lo siguiente: 1) Falta de capacitación docente, administrativa y directiva en los cambios digitales y virtuales; 2) Carencia de efectividad en el diseño, formulación e implementación de políticas públicas; 3) Brechas, asimetrías y disrupciones en las capacidades institucionales para el uso de los medios adecuados para la impartición de educación; 4) Analfabetismo digital por parte de la mayoría de los involucrados 
para hacer uso adecuado de la información; 5) Carencia de un plan estratégico que complemente las asignaturas, los programas y los planes de estudio hacia un futuro digitalizado; 6) Generación de mitos a raíz de la digitalización como un futuro utópico donde las soluciones se encuentran en el uso de los medios virtuales y el internet; 7) Carencia de una conciencia sobre el uso de los canales adecuados que integren, tanto los recursos institucionales como la adecuación de las políticas a los espacios sociales, económicos y sociales; y 8) Ausencia de conscientización sobre una ética constructiva basada en la construcción de plataformas adecuadas al entorno, que permita la generación de propuestas para solventar las problemáticas en lo particular y general (SEP, ANUIES, 2020).

A partir de lo anterior, se puede inferir que existen posibilidades de prever una integración en los espacios educativos de los medios virtuales y digitales que favorezca la correcta inserción de los actores en ellos sin generar las consabidas brechas. Sin embargo, para que ello no sea un elemento de estancamiento o un generador de desigualdad, es necesario un trabajo constante por parte de las instancias involucradas que facilite el correcto desempeño de las y los actores, y donde la institucionalización permita una adecuada ruta hacia un modelo integral con elementos base para ello, mismos que se apoyan en el siguiente esquema como sugerencia de diseño:

Esquema 1. Modelo de integración

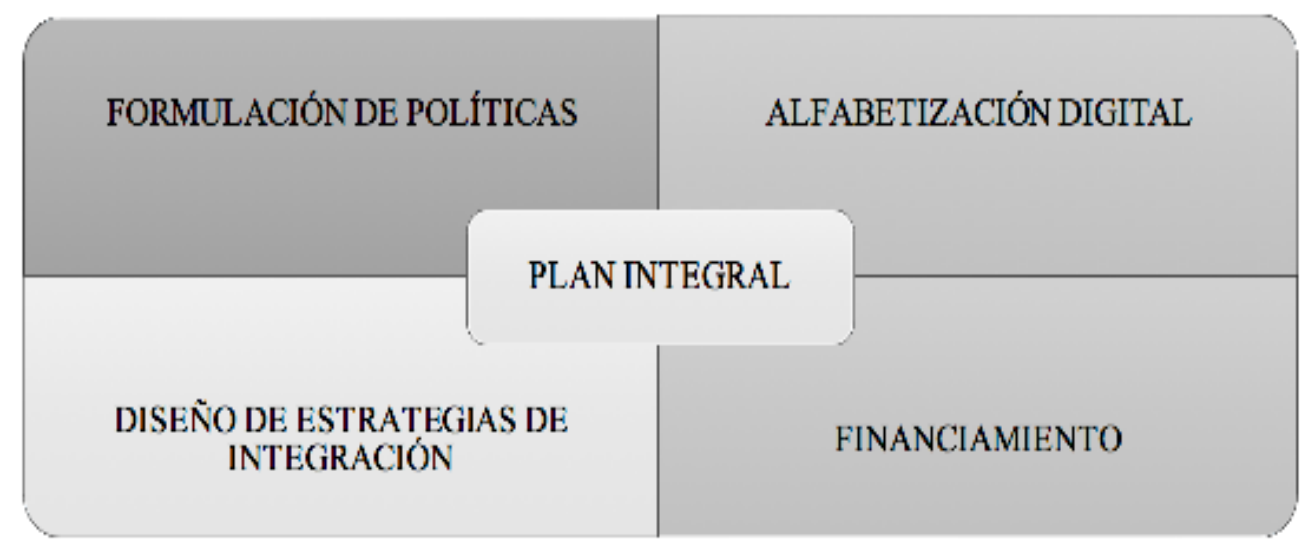

Fuente: Elaboración propia con base en recomendaciones a seguir (2020).

Dados los cambios vertiginosos que siempre son avecinados por los cambios en los esquemas institucionales, el plano actual donde se desenvuelve la educación superior tiene mucha relevancia al tratar de proveer de significado a las decisiones tomadas. En este sentido, para la integración de un espacio de asimilación adecuado, el modelo que se genere debe seguir una lógica en que 
se incluya a los participantes en un sentido de sociabilización y sensibilización (Farías, 2010). Lo anterior sirve como punto de partida para el apartado institucional, y a la vez simboliza el punto crítico y disruptivo ya que plantea el reto de la asimilación, adopción y adaptación de un modus videndi distinto al ya acostumbrado. En la concepción de un futuro formado en la tecnología y los medios virtuales y digitales, el común denominador siempre será la incertidumbre por las disparidades antes comentadas, pero también puede ser el punto en donde la utopía genere la certidumbre sobre los elementos socioculturales que se crean en los entornos de convivencia (Wallerstein, 2016).

De ello se deriva que las políticas públicas e institucionales deben ser diseñadas en el sentido de favorecer el desarrollo constante de los medios que faciliten el uso adecuado de la información, tanto para la toma de decisiones como para la generación de propuestas (Álvarez, 2016). De ahí se deriva que la alfabetización digital tan requerida no se produzca únicamente mediante el proveer de los medios tecnológicos a las personas, sino también de acompañarlas con un diseño de estrategias que permitan acotar la brecha, disminuir las desigualdades y generar mayores oportunidades, tanto para los docentes como para los alumnos, empresarios y sociedad en general (Moreira, Gutiérrez, Vidal, 2012). Ahora bien, el financiamiento requerido debe apoyar a los sectores estratégicos, sin olvidar atender los requerimientos de apoyo sustentable y la apertura de oportunidades para el desarrollo de un futuro en que las universidades retomen su papel esencial de proveedoras y generadoras de conocimiento que esté a la par de los elementos adecuados (Temple, 2014).

\section{La nueva realidad: comentarios sobre un futuro utópico o distópico}

Todos los elementos que ha traído la contingencia y la llamada nueva realidad muestran diversos espacios para su análisis y reflexión. Tanto en los ámbitos educativos como en los empresariales y públicos, se pueden visualizar diversas posibilidades de un futuro de la educación superior, así como la apertura o cierre de oportunidades para los sectores sociales. El incursionar en un mundo utópico pensando quizás en un pasado que traiga añoranzas es lo que genera en gran medida la incertidumbre sobre lo que vendrá, pero apoya a la imaginación con muchos elementos que configuran la institucionalización de un mito sobre la posmodernidad y la adaptación que tendremos de la misma.

Las afectaciones que ha traído esta fase inesperada y aún no superada de la época actual, ha funcionado como una fórmula en la que se trata de descifrar el momento en que vivimos, lo que hemos pasado y lo que vendrá, como si todos y cada uno de ellos fueran un complemento. Dentro de las IES, se pudo observar que ha sido complicado el lidiar con elementos verbalizados por muchos, pero aplicados por pocos, respecto a la ruptura existente entre los procesos tradicionales y los virtuales. El simbolismo de una universidad en un sentido institucionalizado de la palabra ha caído en controversia ya que muchas veces no se cuenta con los elementos nece- 
sarios para asimilar la situación de contingencia y proveer tanto a sus docentes como alumnos de los medios para continuar con sus clases de forma tal que no afecte el proceso pedagógico.

Otro de los factores que han afectado a las IES en el periodo de contingencia ha sido el uso de las tecnologías como base para el desarrollo de las clases y de las plataformas digitales como herramienta en la implementación de herramientas virtuales. El uso de los medios virtuales por parte de las universidades indica la necesidad de poseer la infraestructura necesaria que les permita funcionar de forma remota sin generar conflictos administrativos, operativos $\mathrm{y} / \mathrm{o}$ financieros. En este caso, la inserción de alternativas que permitan crear un cambio que se institucionalice requiere algunas alternativas que generen una visión integral sobre el futuro de la educación en sus diferentes niveles, ya que desde el sustento en el nivel básico se requiere la conceptualización y el desarrollo de habilidades digitales. El cambio efectivo puede significar que las competencias en un futuro modifiquen los esquemas sociales trasgrediendo el sentido de una utopía educativa y creando un futuro donde las alternativas no sean las más adecuadas, donde las brechas sociales y económicas se acrecienten generando con ello una disparidad, desigualdad y segregación.

Si a lo anterior le añadimos el factor social, siendo México un país de contrastes donde la mayoría de las personas aún no viven bajo el mismo esquema de digitalización, podemos prever un futuro en el que las desigualdades aumenten dadas las desproporciones en los espacios económicos. Los sectores rurales, que podríamos decir se encuentran en la desatención constante de los aspectos políticos y sociales, llevan un peso significativo si se trata de encontrar una posible solución sobre el tema. Aunado a ello, las comunidades indígenas o aquellas de usos y costumbres serían como un eslabón perdido en la cadena de sociedad futura integrada por la virtualidad y las TIC.

Se puede inferir, por lo tanto, que en un mundo donde desde hace miles de años nos basamos en los mitos, la integración de la era digital y la tecnología a la vida cotidiana puede tener muchas aristas para analizar. Una de ellas, pensada en un mundo futuro donde existan las mismas oportunidades para todas y todos y no exista la desigualdad, donde la tecnología esté al alcance de todas y todos, y donde exista una paridad como una apoteosis. Otro, donde lo anterior se convierta en un camino disruptivo que produzca un futuro no deseado o no previsto (por algunos) donde las barreras se acrecienten y los complejos cambios hacinen a una gran parte de la población bajo el espectro de la posmodernidad que no se pueda superar o alcanzar.

El mito entonces se convierte en una realidad latente que nos observa como si de ello dependiera el futuro y como si con ellos tratáramos de explicar lo incomprensible. Se debe prestar atención a la forma en que las instituciones generan los próximos mitos con los cuales afrontar la nueva realidad y con los cuales comenzar a construir una posibilidad de educación. Quedan varias preguntas al aire, así como la posibilidad de concretizar diversos análisis a partir de la premisa de los cambios tecnológicos y su afectación a los planos pedagógicos, estructurales, curricula- 
res y gestores en las IES. Las utopías y distopías nos permiten entonces situarnos en una realidad a la cual podemos abordar en aras de recrear las posibilidades que vendrán para la educación post-pandemia.

\section{Referencias}

Aboites, H. (2010). La encrucijada de la universidad latinoamericana. En R. Leher (comp.). Por una reforma radical de las universidades latinoamericanas. Argentina: CLACSO, 95-120.

Acosta-Silva, A. (2013). Políticas, actores y decisiones en las universidades públicas en México: Un enfoque institucional. Revista de la Educacion Superior, 43(165), 83-100.

Aguilar, J. L.; A. Ramírez; R. López (2014). Literacidad digital académica de los estudiantes universitarios: Un estudio de caso. Revista Electrónica de Investigación y Docencia, (11), 123-146.

Álvarez, G. (2016). La ANUIES y la construcción de políticas de educación superior 1950-2015, MéxiCO: ANUIES.

Ansell, B. W. (2008). University Challenges: Explaining Institutional Change in Higher Education. World Politics, 60(02), 189-230. https://doi.org/10.1353/wp.0.0009

Araujo, J. C. (2019). An Indicator of online Collaborative Learning. Revista de Educación Mediática y TIC, 8(1), 171-200. https://doi.org/https://doi.org/10.21071/edmetic.v8i1.11104

Bauman, Z. (2017) Retrotopía. España: Paidós

Bringle, R. G.; P. H. Clayton (2020). Integrating Service Learning and Digital Technologies: Examining the Challenge and the Promise. RIED. Revista Iberoamericana de Educación a Distancia, 23(1), 43-65. https://doi.org/10.5944/ried.23.1.25386

Cady, D.; M. Olson; P. Shea; J. M. Grenier (2011). A Practical Model and Assignments for Using Virtual Worlds in Higher Education. En Hinrichs, R.; C. Wankel, (eds.). Transforming Virtual World Learning (Cutting-Edge Technologies in Higher Education, 4, 87-112. Emerald Group Publishing Limited, Bingley. https://doi-org.wdg.biblio.udg.mx:8443/10.1108/S20449968(2011)0000004009

Castoriadis, C. (2013) La institución imaginaria de la sociedad. España: Tusquets.

Coll, C.; T. Mauri; J. Onrubia (2008a). La incorporación de las TIC a la educación: del diseño tecnopedagógico a las prácticas de uso. En Coll, C.; C. Monereo (eds.) Psicología de la educación virtual. Enseñar y aprender con las tecnologías de la información y la comunicación, España: Morata, 74-103.

Constantino, G.; G. Álvarez (2010). Conflictos virtuales, problemas reales. Caracterización de situaciones conflictivas en espacios formativos online. Revista Mexicana de Investigacion Educativa, 15(44), 65-84. 
Czernik, D. (2006). Alfabetos y saberes: la alfabetización digital. Comunicar: Revista Científica lberoamericana de Comunicación y Educación, (26), 78-82.

De Laat, M.; V. Lally; L. Lipponen; R. Simons (2007). Online Teaching in Networked Learning Communities: A Multi-method Approach to Studying the Role of the Teacher. Instructional Science, 35, 257-286.

Dirección General de Educación Superior Universitaria (2020). DGESU. https://www.dgesu.ses. sep.gob.mx/Indice.html

Eliade, M. (1992). Mito y realidad (II). España: Labor.

Elster, J. (2010). La explicación del comportamiento social: más tuercas y tornillos para las ciencias sociales, España: Gedisa

Facundo, A. (2004) La virtualización desde la perspectiva de la modernización de la educación superior: consideraciones pedagógicas RUSC. Universities and Knowledge Society Journal, $1(1), 1-17$.

Farías, G. (2020) Espacios de aprendizaje en educación superior: de la profesionalización a la innovación para la transformación social, Apertura, 2(2), 18-27

Fernández-Lamarra, N. (2010). Hacia una nueva agenda de la educación a distancia. México: Universidad de Guadalajara.

García, J.; M. Ruiz (2020). Aprendizaje-servicio y tecnologías digitales: un desafío para los espacios virtuales de aprendizaje (Service-Learning and Digital Environment of Learning: Innovative Challenges for Higher Education). RIED. Revista Iberoamericana de Educación a Distancia, 23(1), 31-42. https://doi.org/10.5944/ried.23.1.25390

García, L. (2011). Perspectivas teóricas de la educación a distancia y virtual. Revista Española de Pedagogía, 69(249), 255-272

Gómez, R. R. (2014). Educación superior y transiciones políticas en México. Revista de la Educacion Superior, 43(171), 9-36. https://doi.org/10.1016/j.resu.2014.08.001

Grediaga-Kuri, R. (2011). Revelancia y complejidades del análisis de políticas públicas en educación. Revista Mexicana de Investigación Educativa, 16(50), 679-686.

Guillén, J. A. (2017). Evaluación del aspecto pedagógico de una plataforma virtual: Aplicación de un modelo en la Universidad Autónoma de Santo Domingo (UASD), República Dominicana. Tesis doctoral. España: Universidad de Salamanca.

Hernández, R. (2017). Impacto de las TIC en la educación: Retos y Perspectivas. Propósitos y Representaciones, 5(1), 325-347. https://doi.org/10.20511/pyr2017.v5n1.149

Instituto Federal de Telecomunicaciones (2020). IFC. http://www.ift.org.mx/

Instituto Nacional de Estadísticas y Geografía (2020). INEGI. https://www.inegi.org.mx/temas/ ticshogares/

Kent, R. (2016). Ciclos y avatares de la educación superior en México. México: ANUIES.

Diálo@os 
Leckey, J.; M. McGuigan (1997). Right Tracks-Wrong Rails: The Development of Generic Skills in Higher Education. Research in Higher Education, 38(3), 365-378. www.jstor.org/stable/40196301

Lipovestsky, G. (2000). La era del vacío. Ensayo sobre el individualismo contemporáneo. España: Anagráma.

López, R.; O. González; O. Mendoza; J. Pérez (2009). The Role of the Presidents in the Governance of Mexican Public Universities. México: ANUIES, 1-(29).

López-Segrera, F. (2016). Educación superior comparada: tendencias mundiales y de América Latina y el Caribe. Avaliação: Revista da Avaliação da Educação Superior, 21(1), 13-32.

March, J. G. (1994) A Primer on Decision Making. How decisions Happen. USA: Free Press.

March. J. G.; J. Olsen (1997). El redescubrimiento de las instituciones. La base organizativa de las políticas. México: FCE.

Marúm, E. (2011). Calidad en el servicio en la educación a distancia. Una perspectiva desde México. Revista Iberoemericana de Educación a Distacia, 14(2), 49-62.

Mejía, P. (2014). Cambios y tensiones en la educación superior pública. Reencuentro, (69), 10-21. https://www.redalyc.org/pdf/340/34031038002.pdf

Meyer, J. W.; B. Rowan (1977). Institutionalized Organizations: Formal Structure as Myth and Ceremony. American Journal of Sociology, 83(2), 340-363. https://doi.org/10.1086/226550

Moreno-Rodríguez, D. (2008). Alfabetización digital: el pleno dominio del lápiz y el ratón. Comunicar, 15(30), 137-146. https://www.redalyc.org/articulo.oa?id=158/15811864022

Organización para la Cooperación y el Desarrollo Económico (2020). OCDE. https://www.oecd. org/sti/broadband/broadband-statistics/

Pérez, R.; E. Mena; M. Pereida (2019). Las tecnologías de la información y comunicación como soporte flexibilizador en el proceso enseñanza-aprendizaje. Revista de Educación y DesarroIlo, 50, 59-72.

Popadiuk, S.; E. Rivera; W. Bataglia (2014). Heterogeneity of Isomorphic Pressures: Intertwining the Resource-Based View and the Neoinstitutional Approach. Brazilian Administration Review, 11(4), 455-475. http://dx.doi.org/10.1590/1807-7692bar2014130003

Powell, W.; P. DiMaggio (1999). El nuevo institucionalismo en el análisis organizacional. México: FCE.

Preradovic, N. M.; W. Stark (2019). Identified service learning practices in European higher education. En Aramburuzabala, P.; L. Mcllrath; H. Opazo (eds.). Embedding Service Learning in European Higher Education: Developing a Culture of Civic Engagement. Routledge. 109-131

Rama, C. (2018) Políticas, tensiones y tendencias de la educación a distancia y virtual en América Latina. Argentina: UCSL.

Rangel, A. (2015). Competencias docentes digitales: propuesta de un perfil. Píxel-Bit. Revista de Medios y Educación, (46), 235-248. https://doi.org/10.12795/pixelbit.2015.i46.15 
Rodríguez, D. (2008). Alfabetización digital: el pleno dominio del lápiz y el ratón. INEC, (2014). Estadísticas y Censos. ttps://www.redalyc.org/articulo.oa?id=158/15811864022

Rodríguez, A.; E. Chavez (2020). Cibernética educativa, actores y contextos en los sistemas de educación superior a distancia. Sophia-Colección de Filosofía de la Educación, 23(28), 129 148. https://doi.org/10.17163/soph.n28.2020.04

Rodríguez, I.; A. Meseguer (2018). Immersive Experiences in Online Higher Education: Virtual Presence and Flow. Visvizi, A.; M. D. Lytras; L. Daniela (eds.). The Future of Innovation and Technology in Education: Policies and Practices for Teaching and Learning Excellence (Emerald Studies in Higher Education, Innovation and Technology). Emerald Publishing Limited, 187202. https://doi-org.wdg.biblio.udg.mx:8443/10.1108/978-1-78756-555-520181014

Sandia-Saldivia, B.; J. Montilva (2020). Tecnologías digitales en el aprendizaje-servicio para la formación ciudadana del nuevo milenio. RIED. Revista Iberoamericana de Educación a Distancia, 23(1), 129-148. https://doi.org/10.5944/ried.23.1.24138

Salinas, J. (2004). Campus Extens: Estrategias de educación flexible para la enseñanza universitaria en la Universitat de les illes Balears (UiB). En Sangrà, A.; M. González (coords.). La transformación de las universidades a través de las TIC: discursos y prácticas. Barcelona: UOC, 127-140.

Santos, M. Á.; I. Mella; A. Sotelino-Losada (2020). Movilidad y TIC en aprendizaje-servicio: perspectivas para una sociedad global y tecnológica. RIED. Revista Iberoamericana de Educación a Distancia, 23(1), 67-84. https://doi.org/10.5944/ried.23.1.24180

Secretaría de Educación Pública, Asociación Nacional de Universidades e Instituciones de Educación Superior (2020) Acciones realizadas por las Instituciones de Educación Superior para mantener los servicios educativos ante el COVID-19, así como para contribuir a la salud y bienestar de la sociedad. México: SEP-ANUIES. https://www.tecnm.mx/archivos/slider/ANUIES SEP 15junio2020 V3.pdf

Serra, F.; J. Roca-Piera (2020). Movilidad virtual , reto del aprendizaje de la educación superior en la Europa 2020. RED. Revista de Educación a Distancia, (26), 1-16, https://www.redalyc.org/ articulo.oa?id=54718738001

Silva, Q.; J. Lázaro; A. Miranda; R. Canales (2018). El desarrollo de la competencia digital docente durante la formación del profesorado. Revista de Ciencias Humanas y Sociales, 34(86), 423449. https://dialnet.unirioja.es/servlet/articulo?codigo $=7338181$

Sinisterra, M. (2005). La virtualidad en procesos educativos frente a retos sociales: el analfabetismo. Entramado, 1(2), 92-106. https://www.redalyc.org/pdf/2654/265420471008.pdf

Temple, P. (2014). Universities in the Knowledge Economy. Higher Education Organisation and Global Change. London: Routledge.

Vargas, A. (2015) Literacidad crítica y literacidades digitales: ¿una relación necesaria? (Una aproximación a un marco teórico para la lectura crítica) Revista Folios, (42), 139-160 
Wallerstein, I. (2016) El mundo desencajado. Interpretaciones histórico-mundiales de las continuas polarizaciones, 1500-2000. México: Siglo XXI.

Wilkins, A. (2019). Assembling Schools as Organisations: on the Limits and Contradictiosn of Neoliberalism. The sage handbook of school organization, 509-523. 